\title{
Poleward energy transport: is the standard definition physically relevant at all time scales?
}

\author{
Minyi Liang ${ }^{1} \cdot$ Arnaud Czaja $^{1} \cdot$ Rune Graversen $^{2} \cdot$ Remi Tailleux $^{3}$
}

Received: 14 March 2016 / Accepted: 2 May 2017 / Published online: 17 May 2017

(C) The Author(s) 2017. This article is an open access publication

\begin{abstract}
Poleward energy transport in the atmosphere and oceans constitutes an important branch of the global energy budget, and its role in the climate system has been the subject of many studies. In the atmosphere, the transport is affected by "eddies" and large scale meridional cells, both with zero net mass transport across latitude circles, but also partly by processes associated with a net transport of mass across latitude circles. The latter must cease to operate in steady state, but they may be significant when time variability of the heat budget is considered. Indeed, examination of reanalysis data on short (daily to monthly) timescales shows that mass variations on these timescales result in surprisingly large fluctuations (in excess of $10^{15} \mathrm{~W}$ $=1 \mathrm{PW}$ ) in the poleward heat transport. These fluctuations are referred to as "extensive", for they primarily alter the mass integrated energy of the region considered, but not its averaged value. It is suggested that extensive fluctuations mask more meaningful climate signals present in the heat transport variability on monthly and interannual timescales, and a new formulation is proposed to isolate the latter. This new formulation is applied successfully to reanalysis data and climate model simulations.
\end{abstract}

Keywords Heat transport - Climate variability

Minyi Liang

m.liang14@imperial.ac.uk

1 Department of Physics, Imperial College London, London, UK

2 Department of Physics and Technology, University of Troms $\varnothing$, Troms $\varnothing$, Norway

3 Department of Meteorology, University of Reading, Reading, UK

\section{Introduction}

In the time mean, the atmosphere carries heat meridionally by moving poleward air parcels with high moist static energy and moving equatorward air parcels with low moist static energy (e.g., Held 2001). In midlatitudes, these motions are predominantly associated with traveling cyclones and stationary waves (Oort and Peixóto 1983). At low latitudes, a more complicated set of circulations is involved (Heaviside and Czaja 2013). The resulting time mean poleward heat transport has a simple latitudinal dependence, being weak at the equator and the poles, and peaking near $40^{\circ}$ of latitude in both Hemispheres with a value $\approx 4.5 \mathrm{PW}$ where $1 \mathrm{PW}=10^{15} \mathrm{~W}$ (e.g., Trenberth and Caron 2001).

The need to satisfy mass balance in reanalysis data when computing poleward heat transport is a well known issue which has been addressed in many studies (e.g., Trenberth 1991; Trenberth et al. 2001; Graversen et al. 2007). As an illustration of this point, we display in Fig. 1a, the non mass corrected poleward heat transport from the ERA interim data set to be described more fully in Sect. 2 below. Four curves are shown, each indicating the long wintertime mean values (1979-2014, December through February) at four different times of the day. The spread between these curves is astonishing. For example, at $45^{\circ} \mathrm{N}$, the difference between 0000 and 0600 UTC can be as large as 4 $\mathrm{PW}$, i.e., on the order of the maximum mentioned in the previous paragraph. Surprisingly, we found that even after using the mass correction described in Graversen et al. (2007) and others (e.g., Trenberth 1991), very large differences remain between different times of the day, the typical spread between the curves being now on the order of $1 \mathrm{PW}$ (Fig. 1b). 


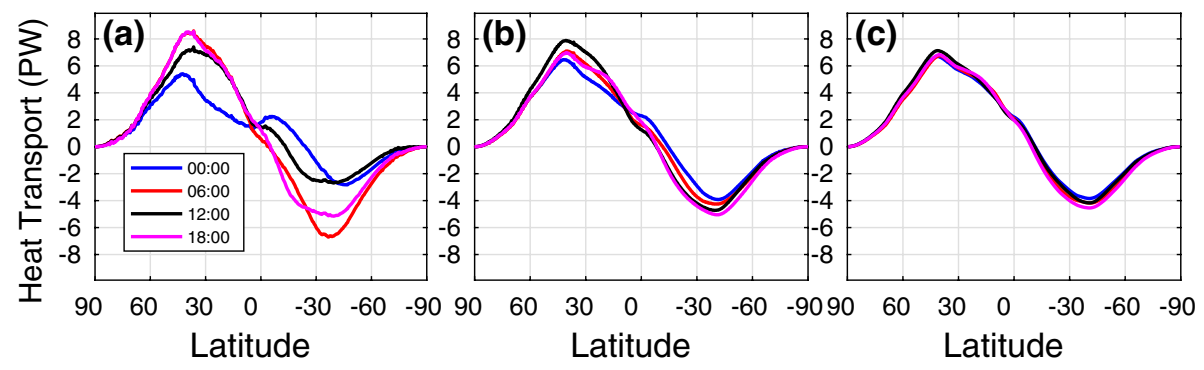

Fig. 1 Boreal winter (DJF) mean poleward heat transport at specific times of the day $(0000,0600,1200,1800$ UTC-see color coding in the subpanel in a) for the period 1979-2014, in units of PW. a As it appears in ERA-Interim reanalysis; $\mathbf{b}$ after a mass correction has been

This finding is puzzling and rationalizing it is our goal in this study. We emphasize that such exercise is not purely of academic interest since the analysis of the time variability of the poleward heat transport on timescales ranging from monthly to decadal can shed light on issues as varied as ocean-atmosphere coupling (Bjerknes 1964; Marshall et al. 2001), the structure of the atmospheric storm track (Messori and Czaja 2013; Novak et al. 2015) and annular modes of variability (Thompson and Barnes 2014). By considering the heat budget of a polar cap, we show below that the large fluctuations evident in Fig. 1a, b are caused by non zero fluctuations in the net (i.e., zonally and vertically averaged) mass transport across latitude circles. We argue that the latter drive changes in the mass integrated energy of a region, but not in the average energy of that region. This consideration allows us to define "extensive" fluctuations in poleward heat transport and also to remove them from a given heat transport record. We argue that the resulting new formulation of the heat budget is more likely to capture meaningful climate signals (e.g., stronger link between heat transport and heat content changes, stronger signals from modes of climate variability such as El Niño, etc.).

The paper is structured as follows. After describing succinctly the ERA interim dataset and a state-of-the-art climate model in Sect. 2, we discuss in Sect. 3 the traditional formulation of the heat transport and its physical interpretation. This leads us to highlight some issues with the standard definition and to propose a new one. A comparison of heat transport variability in both definitions is then carried out in Sect. 4, while further analysis of some features of heat transport in the new definition is given in Sect. 5. A discussion and a conclusion are offered in Sects. 6 and 7, respectively.

\section{Datasets}

The reanalysis ERA-Interim from the European Center for Medium-Range Weather Forecasts (ECMWF) has been applied and using the standard definition of heat transport. $\mathbf{c}$ Using the new definition of heat transport. In the notations of Sect. 3, b, c correspond to $H$ and $H^{\star}$, respectively

used in this study (Dee et al. 2011). Six-hourly (0000, 0600 12001800 UTC) fields from 1979 to 2014 are considered. Note that only winter and summer seasons are taken into account in the analysis, in other words, 36 DJF (December, January and February) and 36 JJA (June, July and August) are provided. The results to be discussed below are only displayed for the DJF period but we found that they also apply to the JJA season.

For quantities using the mass correction, we use the procedure described in detail in Graversen (2006) and Graversen et al. (2007). Briefly, the purpose of the mass correction is to minimize the mass residual, which can be obtained by subtracting a barotropic term at each pressure level. Variables used for the calculation of mass and energy transport are extracted directly from the reanalysis and are calculated in model-space hybrid coordinates. They are then extrapolated to a $0.5^{\circ} \times 0.5^{\circ}$ uniform latitude and longitude grid.

The indices of El Niño and the Arctic Oscillation (AO) are obtained from the NOAA Climate Prediction Center. The El Niño index is taken as the area-averaged sea surface temperature in the region bounded by $5^{\circ} \mathrm{N}-5^{\circ} \mathrm{S}$ and $170^{\circ}$ $\mathrm{W}-120^{\circ} \mathrm{W}$. The $\mathrm{AO}$ index is constructed by projecting the $1000 \mathrm{mb}$ height anomalies poleward of $20^{\circ} \mathrm{N}$ onto the leading mode from the Empirical Orthogonal Function (EOF) analysis of monthly mean $1000 \mathrm{mb}$ height. Time period 1979 to 2014 is considered for both indices.

The Community Earth System Model version 1 (CESM; Hurrell et al. 2013) from the National Center for Atmospheric Research (NCAR) is also used in this paper. The model system includes sub-models for the atmosphere, land-surface processes, sea ice, and ocean. For the atmosphere, the Community Atmosphere Model version 5 (CAM5) is applied with a finite-volume dynamical core, 26 vertical layers and a $\sim 2^{\circ}$ horizontal resolution. It is run in a slab-ocean (SOM) configuration, where the ocean part includes a mixed layer with fixed horizontal transports of energy. These transports, often referred to as 
the q-fluxes, are determined from the climatology of an equilibrium run including a full dynamical ocean model (Bitz et al. 2012). The q-fluxes are given as the climatological mean of the imbalance between the ocean mixedlayer energy change, and the energy flux into the ocean across the atmosphere-ocean and ice-ocean interface and from river discharge. The q-fluxes as well as the depth of the mixed layer were based on the climatology from the last 70 years of a 100-year extension of a pre-industrial control run. The SOM model was additionally run with pre-industrial conditions for 85 years and the last 20 years are used for the atmospheric energy transport calculations in this study.

For all analyses in this study, a linear trend over the whole record was removed, although this procedure was found to have little impact on the results. Anomalies were defined empirically by subtracting the mean seasonal cycle (for either daily or monthly data) from each gridpoint's timeseries.

\section{Poleward heat transport and its interpretation}

\subsection{Formal decomposition of the poleward heat transport}

The energy budget of a polar cap, poleward of a latitude $\phi_{o}$ (north of $\phi_{o}$ in the Northern Hemisphere- the case illustrated below-and south of $\phi_{o}$ in the Southern hemisphere) can be written as (Gill 1982):

$S=H+Q_{T}+Q_{S}$,

in which $S$ represents the storage of energy in the cap:

$S=\frac{\partial}{\partial t} \iiint_{\phi>\phi_{o}} E d x d y d P / g$,

$Q_{S}$ and $Q_{T}$ refer to the energy gained by the cap at its lower (Earth's surface) and upper (top-of-the-atmosphere) boundaries, respectively, and $H$ is the poleward transport of energy across $\phi_{o}$ :

$H=\iint_{\phi_{o}} v(E+P / \rho) d x d P / g$.

In these expressions, pressure coordinates have been used, $\rho$ is density, and the energy per unit mass $E$ is the sum of internal, potential $(\Phi)$ and kinetic $(K)$ energies,

$E=c_{v} T+l_{v} q+\Phi+K$,

where $c_{v}$ is the specific heat at constant volume, $T$ temperature, $l_{v}$ is the latent heat of vaporization and $q$ is specific humidity. In practice, the sum $E+P / \rho$ is well approximated by the moist static energy $h$,

$E+P / \rho \approx c_{p} T+l_{v} q+\Phi \equiv h$, in which $c_{p}$ is the heat capacity at constant pressure and the contribution of kinetic energy to the sum has been neglected. ${ }^{1}$

The different mechanisms contributing to heat transport can be made explicit by developing further the expression (3) after making use of (5). To do so, we introduce the notation $(\ldots)=(\ldots)_{o}+(\ldots)^{\prime}$, where $(\ldots)_{o}$ represents the longitudinal and pressure averaging at latitude $\phi_{o}$,

$(\ldots)_{o} \equiv \frac{\iint_{\phi_{o}}(\ldots) d x d P / g}{\iint_{\phi_{o}} d x d P / g}$,

and in which $(\ldots)^{\prime}$ indicates the departure from this zonal and pressure average. After noticing that $\iint v^{\prime} d x d P / g=0$ and $\iint h^{\prime} d x d P / g=0$, this decomposition allows to write

$H=\iint_{\phi_{o}} v^{\prime} h^{\prime} d x d P / g+h_{o} M$,

in which,

$M=\iint_{\phi_{o}} v d x d y d P / g$

is the mass transport across latitude $\phi_{o}$.

The main insight in this study is to reflect on the physical significance of the second term on the r.h.s of (7). From the point of view of the polar cap poleward of latitude $\phi_{o}$, the term $h_{o} M$ reflects the moist static energy brought to the cap by the existence of a net mass transport across it. If we denote by $E^{\star}$ the polar cap averaged total energy, i.e.,

$E^{\star}=\frac{1}{m_{\text {cap }}} \iiint_{\phi>\phi_{o}} E d x d y d P / g$,

where $m_{\text {cap }}$ is the polar cap mass,

$m_{\text {cap }}=\iiint_{\phi>\phi_{o}} d x d y d P / g$,

then the poleward heat transport can be expressed as,

$H=\iint_{\phi_{o}} v^{\prime} h^{\prime} d x d P / g+\left(h_{o}-E^{\star}\right) M+E^{\star} M$.

\subsection{Physical interpretation of the heat transport}

The decomposition (11) highlights three different mechanisms affecting the heat budget of the polar cap (schematized in Fig. 2).

\footnotetext{
1 Although this approximation is made in the text to simplify the derivation, all calculations with reanalysis and climate model data in this paper use the full expression (4) for $E$.
} 
Fig. 2 Schematic of three processes affecting the heat budget of a polar cap, using the decomposition in Eq. (11). Arrows indicate different components of heat transport while the colors on the block arrows indicate energy content. Dots represent air masses

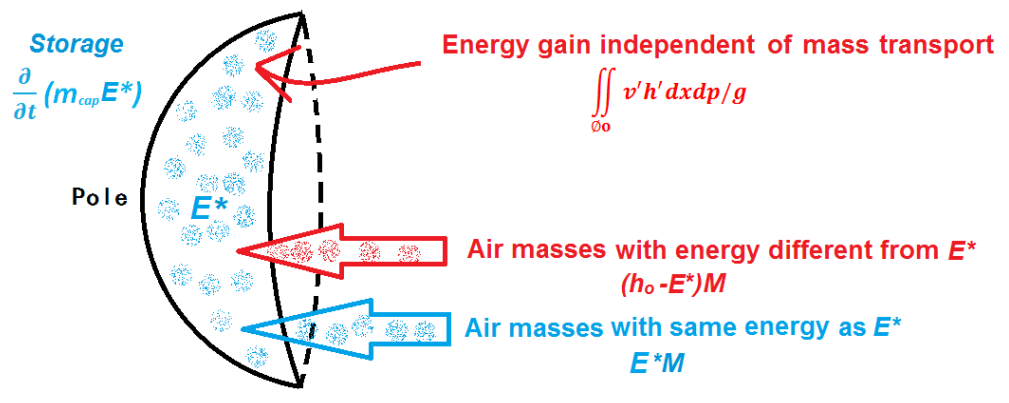

(1) One is to increase the energy of the polar cap without adding any net mass. This is represented by the term $\iint_{\phi_{o}} v^{\prime} h^{\prime} d x d P / g$ on the r.h.s of Eq. (11). It reflects the classic description of poleward heat transport associated with the Hadley circulation in the Tropics (low moist static energy branch moving equatorward at low levels and high moist static energy branch moving poleward at upper levels) and weather systems in midlatitudes (high moist static energy air moving poleward and upward, low moist static energy air moving equatorward and downward).

(2) One is to add mass to the cap by bringing air masses of different energy from that of the cap and/or by doing work on the cap, which is given by the $P / \rho$ term in Eq. (5). The whole process is represented by the term $\left(h_{o}-E^{\star}\right) M$.

(3) Another effect is to add mass to the cap by bringing air masses of same energy. The associated energy gain by this process is $E^{\star} M$. The term $E^{\star} M$ is an extensive quantity, i.e., it increases the energy of the polar cap by simply adding mass at the same energy as $E^{\star}$, but it does not contribute to the increase of the average energy of the cap itself. For this reason we denote the associated fluctuations in heat transport as "extensive".

\subsection{Problems associated with extensive fluctuations in heat transport}

From mass conservation, $M=\partial m_{\text {cap }} / \partial t$ and so when averaging over a long time period, $M \rightarrow 0$. As a result, the contribution of $E^{\star} M$ to the heat transport in Eq. (11) becomes negligible in this limit. ${ }^{2}$ However, on short time scales, and as suggested by Fig. 1, one expects the poleward heat transport to be affected by this term. This is

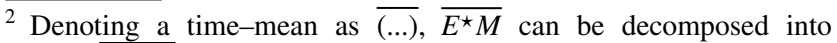
$\overline{E^{\star}} \bar{M}+\overline{E^{\star \prime} M^{\prime}}$. We found that the latter term is two orders of magnitude smaller than the former term in this expression (not shown); when $M \rightarrow 0, \overline{E^{\star}} \bar{M} \rightarrow 0$, and $\overline{E^{\star} M}$ is therefore negligible.
}

readily demonstrated by the existence of a strong linear relationship between fluctuations of $H$ and $E^{\star} M$ at different timescales in Fig. 3 (here considered at $\phi_{o}=0^{\circ}$ in DJF). The squared correlation coefficient generally decreases with averaging time but even for monthly and seasonal averages, values larger than $R^{2}=0.29$ are found. Thus a significant fraction of the variability in seasonal mean poleward heat transport across the equator is purely extensive. Note furthermore that, as will be shown in Sect. 6.1, this effect is not restricted to low latitudes.

There are at least two reasons why one would like to remove the impact of the extensive fluctuations on the heat budget:

1. These fluctuations are exactly balanced by an increase in the energy storage of the cap and do not lead to any climate variability (exactly like adding water to a glass of water at same temperature changes the mass of water in the glass but does not change its temperature). As such, they leave no signature in diabatic effects and do not truly represent a transport of heat: mass at energy $E^{\star}$ is added to the cap at a given time and is removed from it subsequently without interacting with diabatic processes since the mass has the same energy as the cap. As a result there is no net transfer of energy. This is very clearly seen in the large magnitudes of the fluctuations seen in Figs. 1 and 3 which, if they involved changes in radiative or surface fluxes, would require unrealistically large values for these terms. ${ }^{3}$

2. The magnitude of the extensive heat transport fluctuations depends arbitrarily on the choice of a reference state when computing the total energy (e.g., whether counting $\Phi$ from sea level or not and taking Kelvin or Celsius degree as the unit of temperature). For the standard choice used in this study $(\Phi=0$ at sea level and temperature expressed in $\mathrm{K}$ ), the extensive fluctua-

\footnotetext{
${ }^{3}$ For example, the variations of $5 \mathrm{PW}$ seen in Fig. 3a at the equator are equivalent to a spatially uniform imbalance of $\approx 50 \mathrm{Wm}^{-2}$ in $\left(Q_{T}+Q_{S}\right)$ over the whole Northern Hemisphere!
} 
Fig. 3 Poleward heat transport $H$ vs $E^{\star} M$ at the equator based on 36 boreal winters (DJF) from 1979 to 2014 . The data is averaged every $\tau$ days and the value of $\tau$ is given in each panel. $R^{2}$ is the squared correlation coefficient. Blue lines indicate the least square fit for each panel. Note that the limits of $x$-axis and $y$-axis are different for the top and bottom panels. All terms are expressed in PW
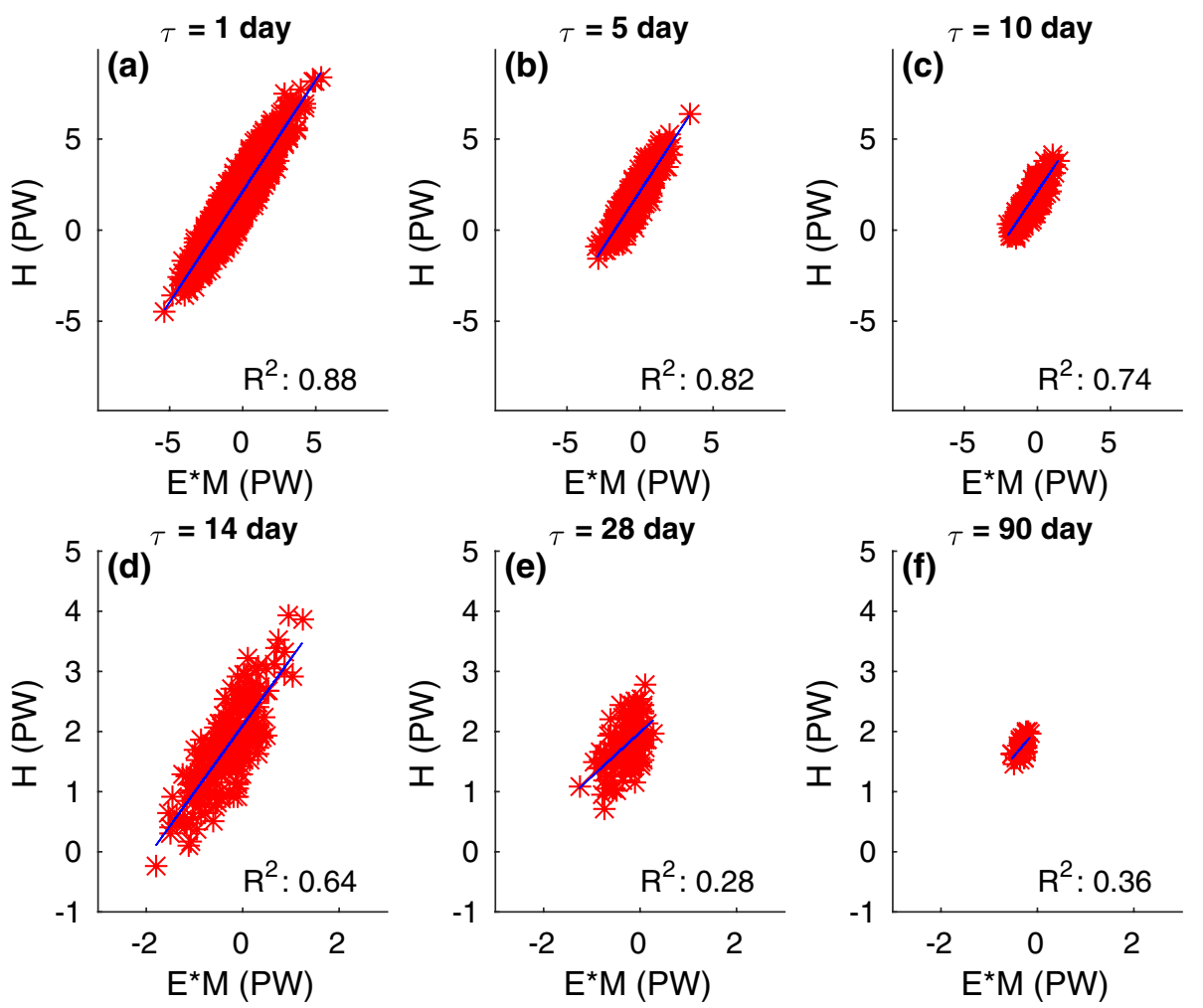

tions are particularly large (see Fig. 1 and the scales in Fig. 3) and are thus likely to mask meaningful climate signals in the heat budget.

For these reasons we propose below to introduce a new definition of the heat transport.

\subsection{A new definition of heat transport}

The discussion in Sect. 3.2 suggests that a simple way to remove the extensive fluctuations in heat transport is to subtract the component $E^{\star} M$ from the poleward heat transport $H$. Indeed, writing the heat content of the polar cap as $m_{c a p} E^{\star}$, the heat budget (1) can be rewritten as:

$m_{\text {cap }} \frac{\partial E^{\star}}{\partial t}+E^{\star} M=H+Q_{T}+Q_{S}$,

Using Eq. (11), we thus obtain,

$m_{\text {cap }} \frac{\partial E^{\star}}{\partial t}=\iint_{\phi_{o}} v^{\prime} h^{\prime} d x d P / g+\left(h_{o}-E^{\star}\right) M+Q_{T}+Q_{S}$,

or, more compactly,

$S^{\star}=H^{\star}+Q_{T}+Q_{S}$,

where we have introduced a new storage term $S^{\star}$ :

$S^{\star}=m_{c a p} \frac{\partial E^{\star}}{\partial t}$, and the new heat transport $H^{\star}$ :

$H^{\star}=\iint_{\phi_{o}} v^{\prime} h^{\prime} d x d P / g+\left(h_{o}-E^{\star}\right) M$,

Note that this is also more simply:

$H^{\star}=H-E^{\star} M=\iint_{\phi_{o}} v\left(h-E^{\star}\right) d x d P / g$.

Although formally similar to (1), the interpretation of the polar cap heat budget in Eq. (14) is different in that it is a budget for the average energy of the polar cap (i.e., $E^{\star}$ ), rather than the mass integrated energy of the polar cap (i.e., $m_{c a p} E^{\star}$ ). The extensive fluctuations in heat transport have been removed, although $M$ still contributes to poleward heat transport through the second term on the r.h.s of (16). Note that the difference $h-E^{\star}$ in Eq. (17) is $h-E^{\star}=E-E^{\star}+(P / \rho)$ which is independent of a reference state for energy since $P$ and $\rho$ are independent of the latter (pressure is uniquely defined as force per unit area and density as mass per unit volume).

The new heat transport was computed from the ERA interim dataset using (17) and (9) at different times of the day over a long (1979-2014) wintertime period. As expected, the resulting diurnal fluctuations are greatly dampened (Fig. 1c) compared to those obtained using the standard heat transport definition in Eq. (3) - see Fig. 1 b. 

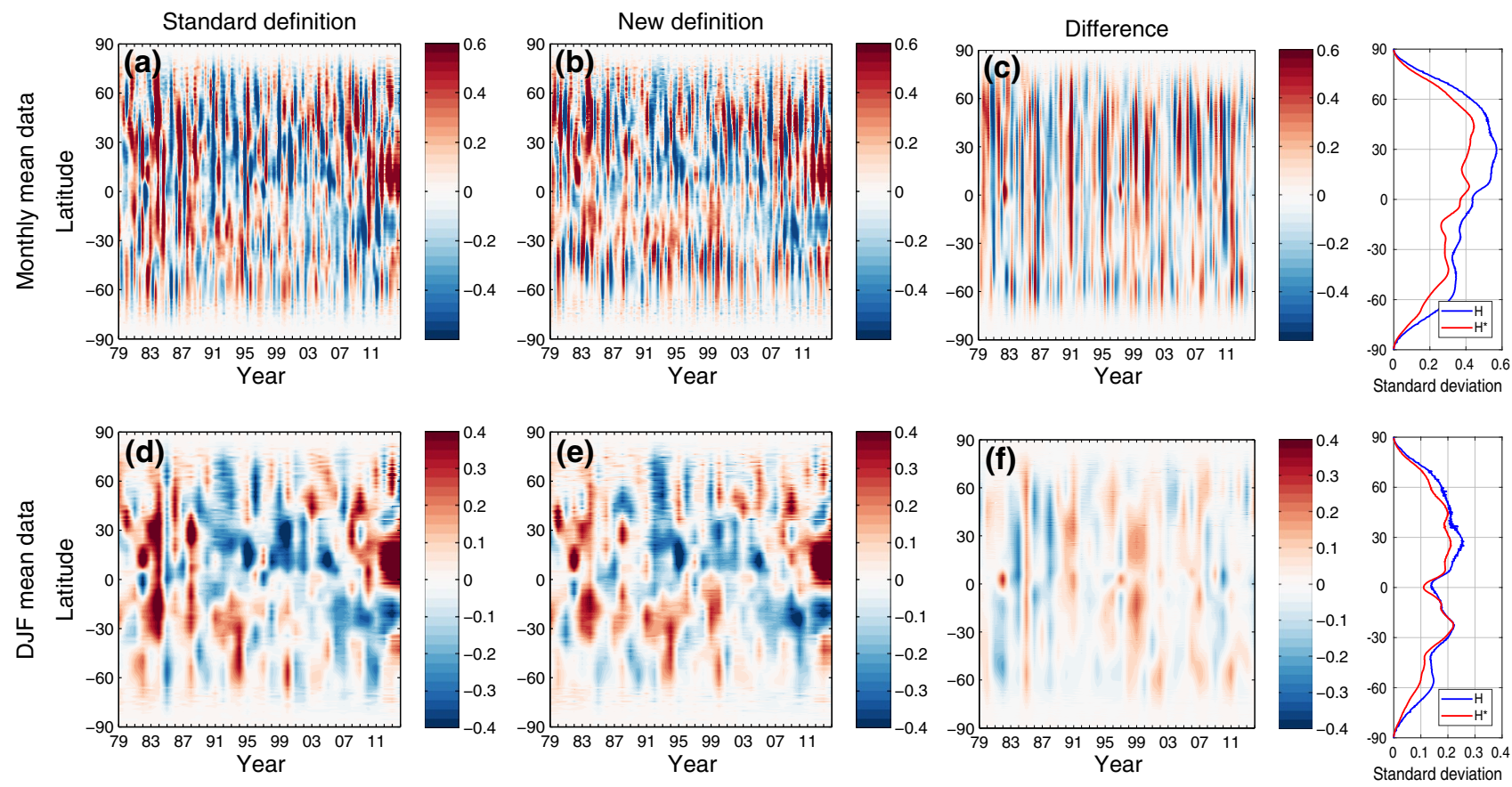

Fig. 4 Latitude-time (in years) diagram of poleward heat transport ( $H$, left column; $H^{\star}$, middle column; the difference $H^{\star}-H$, right column) in PW. For all subplots, the annual cycle calculated over the period 1979-2014 has been removed. The top and bottom panels are calculated using monthly mean data and seasonal (DJF) mean data, respectively. Note that colorbars are different for the top and bottom panels. The far right panels indicate (in $\mathrm{PW}$ ) the standard deviation of $H$ (blue) and $H^{\star}$ (red) as a function of latitude for monthly (upper panel) and seasonal mean data (lower panel)

\section{Comparison of the standard and the new definitions of the poleward heat transport}

\subsection{Temporal and spatial variability}

Time series of heat transport anomaly represented by both monthly mean and DJF mean data are computed with the standard and new heat transport definitions and are displayed in Fig. 4 (note that the annual cycle has been removed to obtain the anomaly for the time period 1979-2014). The panels on the far right side of this figure shows the standard deviation of the monthly (upper panel) and seasonal (lower panel) heat transport anomalies. One sees a larger variability at all latitudes in the monthly data by about $0.1 \mathrm{PW}$ and this increased variability persists for seasonal anomalies in some latitude bands but not all. The timeseries plotted in panels (a, b, d, e) confirm this conclusion, and show evident differences when monthly averaged (Fig. 4c) but less so for the seasonal means (Fig. 4f). This result is consistent with the fact that the contribution of $M$ to $H$ becomes smaller as timescales increase, as was seen in Fig. 3-see also Fig. 10 in Sect. 6 below.

Inspection of Fig. 4 suggests a narrower latitudinal structure of anomalies in $H^{\star}$ compared to those in $H$. For example, a comparison of Fig. 4d, e shows that in 1988, poleward heat transport $H$ (represented by the red area in the figure) extends all the way from $30^{\circ} \mathrm{S}$ to $30^{\circ} \mathrm{N}$, while this feature is not seen in $H^{\star}$. To confirm this visual impression, we next examine the autocovariance of $H$ and $H^{\star}$ in monthly mean data. The result is shown at $10^{\circ} \mathrm{N}$ in Fig. 5 (note that, as for Fig. 4, the annual cycle has been removed to obtain the anomaly for the time period 1979-2014). Besides highlighting the larger autocovariance for $H^{\star}$ compared to that for $H$ (consistent with the larger variability in $H$ than in $H^{\star}$ discussed in the previous paragraph), the resulting figure reveals a change in spatial structure, the autocovariance of $H^{\star}$ (pink curve) displaying a divergence/ convergence mode of heat export/import by the atmosphere at low latitudes (pink curve) which is not seen with that of $H$ (green curve). Note that a similar behavior was observed at other low latitudes, but not poleward of $25^{\circ}$ (there the autocovariance functions decay to zero without changing sign, but it is still narrower with $H^{\star}$ than with $H$-not shown).

Another point of comparison between $H$ and $H^{\star}$ is to estimate years or seasons with large or weak poleward heat transports. This was considered for the $40^{\circ} \mathrm{N}-50^{\circ} \mathrm{N}$ band, which corresponds to the location of the Northern 


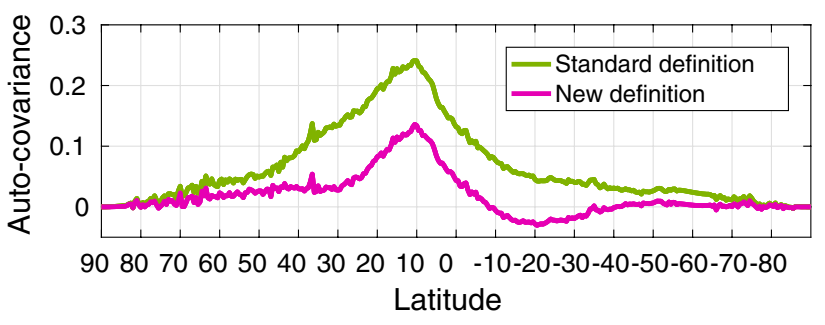

Fig. 5 Autocovariance of monthly anomalies in heat transport at $10^{\circ}$ $\mathrm{N}$ : $H$ (green) and $H^{\star}$ (pink). Units are $\mathrm{PW}^{2}$
Table 1 Boreal winters (DJF) in which heat transport averaged over $40^{\circ} \mathrm{N}-50^{\circ} \mathrm{N}$ reached extremes in the period 19792014, based on the standard and new heat transport definitions

\begin{tabular}{ll}
\hline New definition & $\begin{array}{l}\text { Standard } \\
\text { defini- } \\
\text { tion }\end{array}$ \\
\hline $\begin{array}{l}\text { More heat poleward } \\
1980\end{array}$ & - \\
1984 & 1984 \\
- & 1986 \\
1995 & - \\
2003 & 2003 \\
2009 & 2009 \\
Less heat poleward & \\
1981 & - \\
1987 & - \\
1988 & - \\
1989 & 1989 \\
1993 & 1993 \\
1996 & 1996 \\
1999 & 1999 \\
2007 & 2007 \\
\hline
\end{tabular}

The year reflects that of the December month. The top panel indicates the atmosphere carries more heat poleward than on average; the bottom panel the opposite
Hemisphere storm-track in winter. Boreal winters where the heat transport in this band (i.e., the timeseries of heat transport averaged each winter over the $40^{\circ} \mathrm{N}-50^{\circ} \mathrm{N}$ band) exceeds $+1 /-1$ standard deviation were identified for $H$ and $H^{\star}$. As can be seen in Table 1, although most years are similar when considering the new and standard definition, there are a few differences: for example, year 1981, 1987 and 1988 do not show up in the timeseries associated with the standard definition. Inspection of (16) and (11) suggests that, surprisingly, this must arise because the $v^{\prime} h^{\prime}$ term is not the dominant contributor to the winter-to-winter variability of $H$ (if it were, it would also dominate $H^{\star}$, since $\left|\left(h_{o}-E^{\star}\right) M\right| \ll\left|h_{o} M\right|$ and extreme years would be the same for both heat transport timeseries). Thus the identification of years where the atmosphere carries large or small amount of heat is affected by the presence of extensive fluctuations in the standard definition. This important role of the term $E^{\star} M$ for the standard definition can be clearly seen in Fig. 6, which displays, at different timescales, the standard deviation of the different components in Eq. (11). It is seen that at all timescales $E^{\star} M$ (blue) has a comparable magnitude to that of $\iint v^{\prime} h^{\prime} d x d P / g$ (yellow). Further quantification of the impact of the variability of $M$ on that of $H$ and $H^{\star}$ is postponed to Sect. 6.1.

\subsection{Link to climate patterns of EI Niño and the AO}

To analyze the relationship between heat transport and climate oscillations such as El Niño and the AO, correlation coefficients between those variables for different latitudes are calculated and shown in Fig. 7. The largest correlation coefficients are found at around $50^{\circ} \mathrm{N}$ for AO (Fig. 7a) and around $5^{\circ} \mathrm{S}$ for El Niño (Fig. 7b). In a positive phase of the AO the atmosphere carries less heat poleward $50^{\circ} \mathrm{N}$. Conversely, in a El Niño phase the atmosphere carries more heat poleward at $5^{\circ} \mathrm{S}$.

These correlations support the view that poleward heat transport is linked to modes of climate variability and are consistent with previous findings. For example, El Niño years intensify the Hadley circulation (Oort and Yienger 1996), which subsequently transport more heat from the tropics to higher latitudes (Sun 2000; Sun and Trenberth 1998), and the reverse is true for La Nina years. Likewise, the relationship between the AO (or North Atlantic Oscillation) was studied by Carleton (1988). His Fig. 9 suggests a weaker poleward sensible heat transport in a positive phase NAO (driven by changes in stationary waves) which is consistent with our finding. This overall suggests that the strong zonal circulation found in a positive phase of the NAO limits the capacity of the atmosphere to carry heat poleward. Conversely, in the negative phase, a more wavy Jet Stream appears to enhance the capacity of the atmosphere to carry heat poleward.

The correlations in Fig. 7 have been computed for both the standard (green) and new (magenta) definitions, and they are seen to have larger magnitude with the new definition. Admittedly the differences are small, but interestingly, they are systematic in the region of interest (e.g., northern extra-tropics in Fig. 7a or tropics in Fig. 7b). This suggests that the new definition of heat transport captures better climate signals than the standard definition, as was hypothesized in Sect. 3.3. We return to this point more fully in Sect. 6.2 below. 
Fig. 6 Variability of the different components of the heat transport using the decomposition in Eq. (11). Blue lines are the standard deviation of $E^{\star} M$, orange lines that of $\left(h_{o}-E^{\star}\right) M$, and yellow lines that of $\iint v^{\prime} h^{\prime} d x d P / g$. The four panels represent a 6-hourly b daily $\mathbf{c}$ monthly and d seasonal (DJF) mean data. All calculations are for the time period 1979-2014 with units of PW. Note that $y$-axis limits are different between the top and bottom panels and that all terms have been divided by a factor of 10 to offer easier comparison with Fig. 8
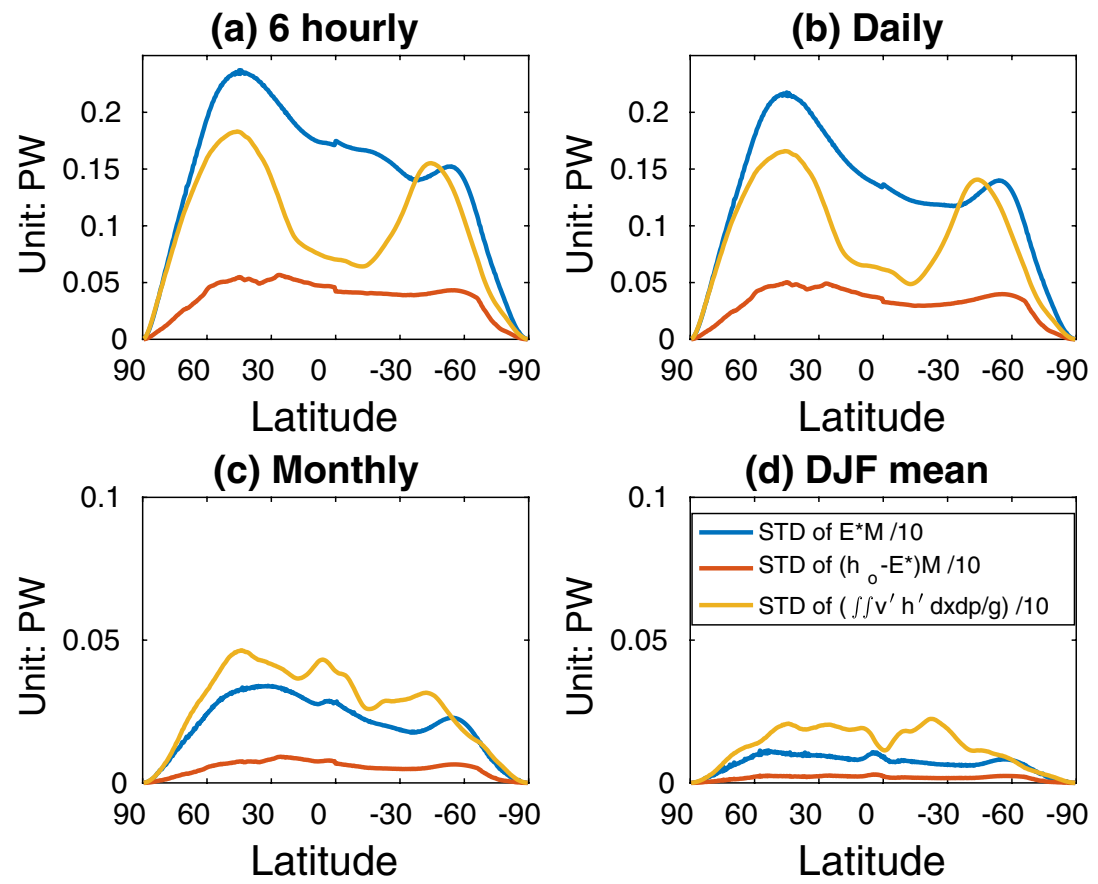

Fig. 7 Temporal correlation between poleward heat transport and $\mathbf{a} \mathrm{AO}$ index; b El Niño index at different latitudes (see Sect. 2 for a definition of these indices). Red lines indicate $95 \%$ significance level assuming statistical independence every winter (AO) or every three winters (El Niño). Negative correlations indicate anomalous southward heat transport
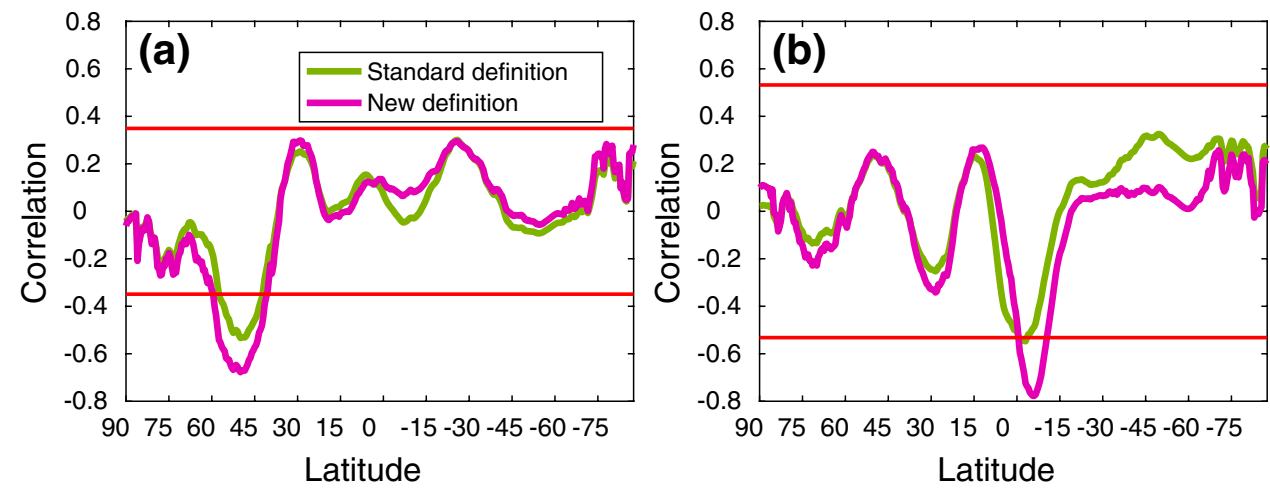

\section{Further investigations}

\subsection{Latitudinal structure of the variability of $H^{\star}$}

The standard deviation of heat transport $H^{\star}$ (Fig. 8, blue curves) shows an interesting dependence on time scales: on 6-hourly and daily time scales, the signature of the extra-tropical storm track is evident (Fig. 8a, b, blue curves), but the tropics dominate the variability on longer time scales (Fig. 8d, blue curves). By using the decomposition (16) we found that these features arise mostly in response to the $v^{\prime} h^{\prime}$ term, the variance of $\left(h_{o}-E^{\star}\right) M$ is very small on 6 hourly and daily timescales and almost negligible on longer time scales (Fig. 6, compare orange and yellow curves). Thus, the large/weak events shown in Table 1 for $H^{\star}$ mostly reflect the larger of weaker magnitude of the $v^{\prime} h^{\prime}$ term. This contrasts with $H$ for which we found in Sect. 4.1 that years with high or low heat transport were also affected by large or weak fluctuations in $M$.

\subsection{Climate model data}

Mass conservation is not satisfied in reanalysis data due to the inherent properties of the data assimilation process. Thus when using reanalysis data, mass correction (Trenberth 1991; Graversen 2006; Graversen et al. 2007) should be carried out before conducting the new heat transport calculation. Nevertheless, heat transport variability in climate models might also be dominated by the extensive fluctuations isolated in Sect. 3, despite the fact that these models conserve mass. To check this, we have repeated the calculation in Fig. 1 with the CESM model (see Sect. 2). The spread among the estimates of the new heat transport at four different times of the day is, again, much smaller with the new definition (Fig. 9b) than with the standard definition (Fig. 9a). This indicates that our 
Fig. 8 As in Fig. 6 but for the variability of the poleward heat transport $H^{\star}$ (blue line) and the difference $H^{\star}-H_{\text {apprx }}^{\star}$ (orange). Note that $y$-axis limits are different between the top and bottom panels (a) 6 hourly

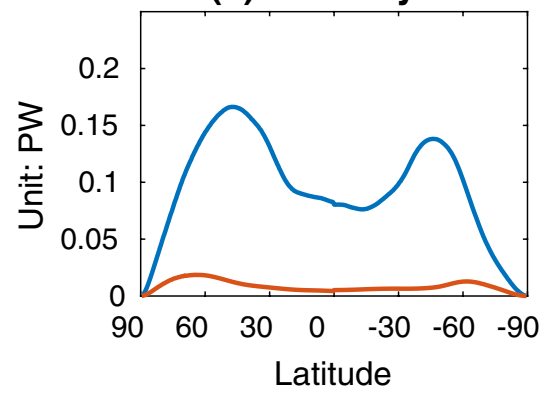

(c) Monthly
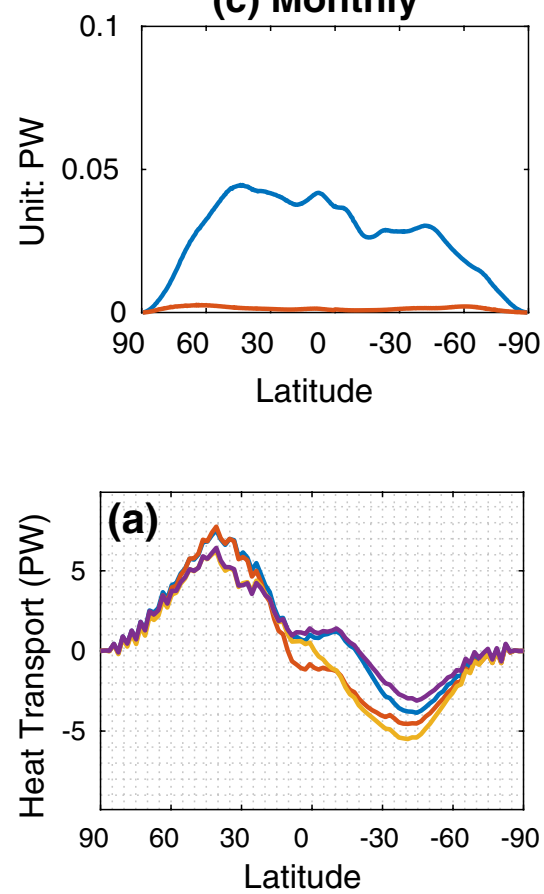

(b) Daily

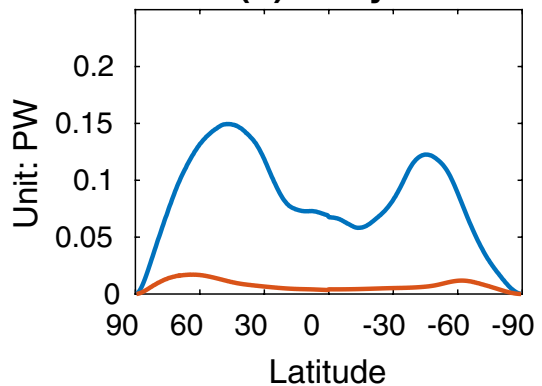

(d) DJF mean
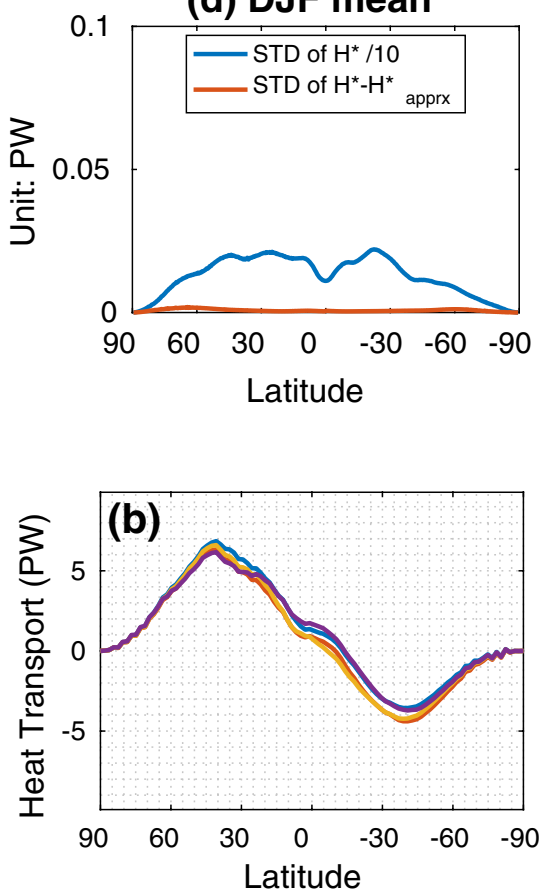

Fig. 9 Same as Fig. 1b, c, but for the CESM model rather then ERA-interim data methodology has relevance to heat transport variability in climate model simulations, as well as in reanalysis data.

\subsection{Practical implementation}

The computation (17) is to some extent complicated by the requirement to compute the time evolution of the $3 \mathrm{D}$ average quantity $E^{\star}$ defined in Eq. (9). However, we found that $E^{\star}$ varies little over both short and long time scales, suggesting that we can instead replace it by its time averaged value (indicated by an overbar):

$\overline{E^{\star}}=\frac{1}{m_{\text {cap }}} \iiint_{\phi>\phi_{o}} \bar{E} d x d y d P / g$.

With this approximation the new heat transport becomes,

$H_{\text {apprx }}^{\star}=\iint_{\phi_{o}} v\left(h-\overline{E^{\star}}\right) d x d P / g$.
We found that the standard deviation of the difference between the time series of the new heat transport $H^{\star}$ and its approximation $H_{\text {apprx }}^{\star}$ is almost negligible, being at most $0.03 \mathrm{PW}$ on 6-hourly and daily time scales and less than 0.005 PW on monthly and inter-annual time scales (see Fig. 8, orange curves).

\section{Discussion}

\subsection{Quantification of the impact of $M$ on $H$}

Standard and new definitions of heat transport are approximately equal when the poleward mass transport can be neglected (see also footnote 2). Indeed, from Eqs. (16) and (11),

$H \approx H^{\star} \approx \iint_{\phi_{o}} v^{\prime} h^{\prime} d x d P / g \quad$ when $M \rightarrow 0$. 
Fig. 10 a Squared correlation $\left(R^{2}\right.$, color $)$ of the linear regression between standard heat transport $(H)$ and mass transport $(M)$, for different length of averaging ( $x$-axis, in days). b Autocorrelation of mass transport $M$ as a function of time lag in days. All calculations were carried out using 36 winter seasons (DJF) from 1979 to 2014 (a)

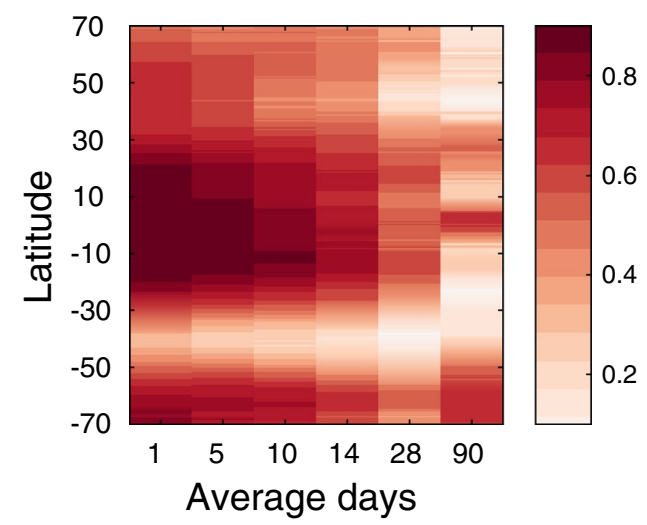

(b)

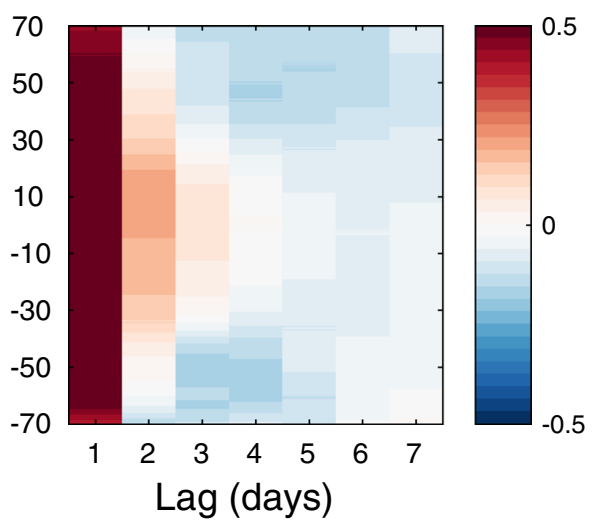

In this section, we examine at which timescale this approximation becomes valid.

To do so we have repeated the analysis in Fig. 3 at all latitudes (Fig. 10). This figure displays the square correlation coefficient $R^{2}$ between timeseries of $H$ and $M$ at all latitudes (y-axis), for varying amount of temporal smoothing (x-axis). The $R^{2}$ values decrease with averaging time at all latitudes but the decay is slow, with $R^{2}$ exceeding 0.3 for the 90-day average timeseries not only at the equator but also in subtropical and high latitudes. Very similar results were found for the JJA period (not shown). We reemphasize that, as all calculations discussed in this paper (except for Figs. 1a, 9), a mass conservation correction has been applied to the data. Despite the latter, Fig. 10 suggests that up to $30 \%$ of the variance of the heat transport at these timescales is reflecting what we referred to as extensive heat transport fluctuations in Sect. 3. Indeed, from Eq. (11), the component of $\mathrm{H}$ linearly dependent on $M$ is $\left(h_{o}-E^{\star}\right) M+E^{\star} M=h_{o} M$, and thus depends on the magnitude of the moist static energy at the latitude considered. As a result, the higher the moist static energy of a latitude band on average, the larger the contribution of $M$ to the fluctuations of $H$. This is consistent with the tongue of largest $R^{2}$ in Fig. 10a being centered on low latitudes. In addition, the timescale at which the contribution $E^{\star} M$ becomes negligible also depends on how fast time averaging can reduce the variability in $M$. For a random process, the variance of the time averaged time series is sensitive to the decorrelation time of the process (e.g., Czaja et al. 2003). We found that indeed, the slower decay in Fig. 10a found at low latitudes is also consistent with a larger decorrelation time of $M$ at these latitudes (shown in Fig. 10b).

For reference, we have repeated the analysis in Fig. 10 with $H^{\star}$ rather than $H$. As expected, we found much weaker correlations (not shown), consistent with a weaker linear dependence $\left(h_{o}-E^{\star}\right) M$ in Eq. (16), and with the small contribution of this term to the variability of $H^{\star}$ displayed in Fig. 6 (compare the magnitude of orange and yellow curves). Note however that $M$ still contributes to the variability of $H^{\star}$, as one might intuitively expect: it is only the extensive component of the heat transport which is removed by our procedure.

\subsection{Which definition is preferable?}

We have shown in Sect. 4 that new and standard definitions of the heat transport differ significantly from one another. Although we have presented conceptual arguments in favour of the new definition in Sect. 3, it is important to assess which definition is better suited to studies of the time variability of the heat budget. As an attempt to do so, we focus on the link between heat transport and heat content. We expect intuitively little correlation between these two on short timescales, as the heat content needs time to build up. On longer timescales though, more poleward heat transport towards the cap is expected to lead to a larger heat content and thus a significant correlation is expected. We ask the question: which definition captures better this relationship?

To address this question, we have computed the temporal correlation between the heat content (HC, simply equal to $m_{\text {cap }} E^{\star}$ using the notations in Sect. 3.4) of a given polar cap and either $H$ or $H^{\star}$ across the bounding latitude of the cap. The result is shown in Fig. 11, and recovers the expected results that weak correlations are found on short timescales (upper panels). On longer timescales (lower panels), we find systematically larger correlations with the new definition of heat transport (red curve) than with the standard one (blue curve), except at high southern latitudes (over Antarctica).

We have also computed the correlations between the two definitions of heat transport and the two storage $S$ and $S^{\star}$ in Eqs. (1) and (14), respectively (not shown). We found stronger correlations between $H$ and $S$ than between $H^{\star}$ and $S^{\star}$, indicating that the term $E^{\star} M$ dominates both the storage of energy and the heat transport in the standard 
Fig. 11 Squared correlation coefficient $\left(R^{2}\right)$ between heat transport and heat content $(\mathrm{HC}$ $=m_{\text {cap }} E^{\star}$ using the notations in Sect. 3, where a southern cap is used at southern latitudes and a northern cap is used at northern latitudes). Blue lines are for correlation between $H$ and $H C$; red lines are for $H^{\star}$ and $H C$. The four panels represent a 6-hourly b daily $\mathbf{c}$ monthly and d seasonal (DJF) mean data, respectively, for the time period 1979-2014
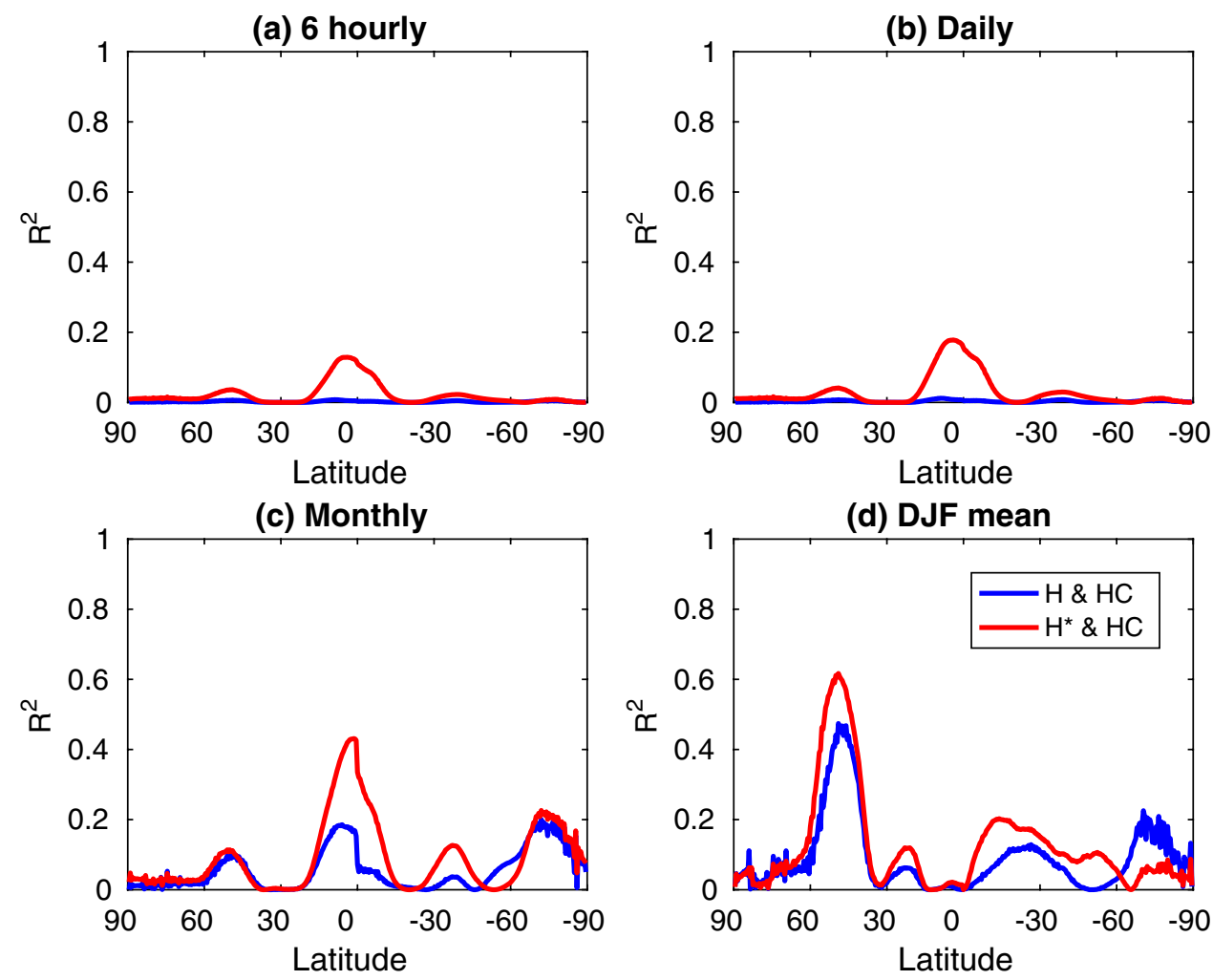

(d) DJF mean

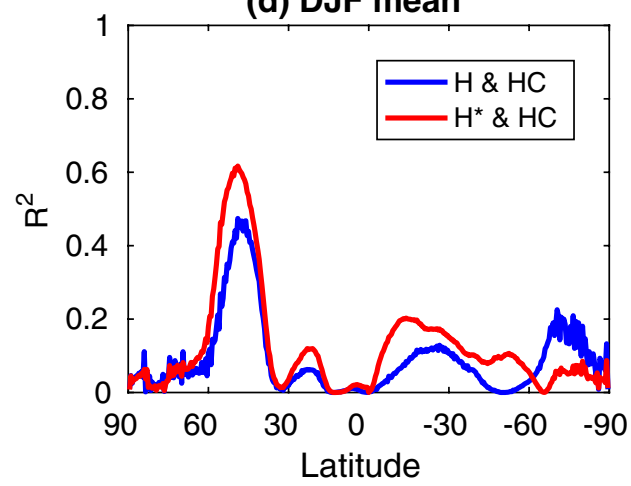

formulation of the heat budget. The weaker correlations obtained between $H^{\star}$ and $S^{\star}$ show that both the diabatic terms $Q_{T}$ and $Q_{S}$ and heat transport $H^{\star}$ are equally important in the new formulation of the energy budget. As longer timescales are considered, both $H$ and $H^{\star}$ become comparable and both must oppose the diabatic terms in the heat budget. On shorter timescales however, the new formulation captures a finer balance in which both storage, transport and diabatic effects are all important.

We would also like to emphasize that the issue highlighted in this study is only relevant to the variability of the total heat transport. It is not relevant to that of the heat transport driven by transient or stationary eddies, or overturning circulations (e.g., Peixoto and Oort 1992) since all these are associated, by construction, with no net mass transport across latitude circles (i.e., $M=0$ for all these motions and they are all included in the $v^{\prime} h^{\prime}$ term in Fig. 2).

\subsection{Domain dependence}

The new heat transport definition was phrased in the context of the heat budget of a cap poleward of a given latitude $\phi_{o}$. The calculation of $H^{\star}$ at $\phi_{o}$ was based on a cap with latitude $\phi \geq \phi_{o}$ for $\phi_{o} \geq 0$ (Northern Hemisphere) and a cap with $\phi \leq \phi_{o}$ for $\phi_{o}<0$ (Southern hemisphere). The resulting Eq. (14) is well posed and offers a straightforward analysis of the heat budget of that cap. Nevertheless, it is important to emphasize that there is an arbitrariness in the choice of the cap. For $\phi_{o}>0$ for example, we could also choose a very large cap defined by latitudes south of $\phi_{o}$ and this would lead to a different heat transport $H^{\star}\left(\phi_{o}\right)$ since, in general, the average energy $E^{\star}$ of the northern and southern caps differs. Although in practice we found the differences to be very small (timeseries of heat transport at different latitudes with both choice of caps were found to be virtually indistinguishable - not shown), more work needs to be carried out to assess whether a different methodology could alleviate this caveat.

\section{Conclusion}

The standard definition of poleward heat transport was found to lead to surprisingly large fluctuations on timescales shorter than a few years. For example, it can change dramatically by $4 \mathrm{PW}$ in just $6 \mathrm{~h}$ in ERA interim reanalysis, which is of the order of the maximum time mean poleward heat transport $(\approx 4.5 \mathrm{PW})$. This unrealistic behavior might at first be thought to result from issues with mass conservation in reanalysis data. However, even after adjusting the mass balance, the problem was shown to remain.

We have argued in this paper that this problem arises because of the dominance of mass transport related fluctuations in heat transport which do not affect the average energy of a region, but affects the mass integrated energy of the region. These extensive fluctuations, we have 
suggested, are not related to climate signals (e.g., temperature), nor do they represent a true heat transport. In addition, they are dependent on an arbitrary choice of reference for energy. Nevertheless, by using linear regression analysis we have estimated that typically a third of the variance in heat transport on monthly and inter-annual (i.e., DJF averaged) timescales reflect directly these extensive fluctuations in the ERA interim dataset (based on the $R^{2}$ values in Fig. 10). Thus, we clearly answer by the negative to the question raised in the title of our study.

A new definition of heat transport was introduced to address this problem in the context of the total energy $(E)$ budget of an hemispheric cap, poleward of a given latitude $\phi_{o}$. The idea is to subtract an instantaneous cap-averaged energy $E^{\star}$ from $E$ in the standard heat transport formulation. In doing so one shifts from a consideration of the budget for the mass integrated energy of the cap into a consideration of the budget for the average energy of the cap. In order to simplify the calculation, an approximation to the new definition was also proposed, and no significant difference was found between the exact and approximate formulations. The formulation was also shown to produce similar results in a state-of-the-art climate model simulation, indicating that the approach is not limited to reanalysis datasets.

The new heat transport definition was shown to lead to stronger correlations with heat content than the standard one, and was also shown to display a more robust statistical link with modes of climate variability. For example, a clearer relationship between the $\mathrm{AO}$ and heat transport emerges in our analysis, the atmosphere carrying less heat poleward in a positive phase than in a negative phase of the AO. El Niño variability was also found to be linked more robustly to changes in poleward heat transport with more poleward heat transport at low southern latitudes in El Niño years. New and standard definitions of heat transport also display pronounced differences in autocovariance functions and produce different time sequence of events with large/ weak poleward heat transport. Finally, the variability of the heat budget in the new formulation is also different, with a larger role for diabatic effects, even on short (less than seasonal) timescales. It is hoped that the new definition of heat transport may shed further light on the intuitive link between energy transport and climate variability.

Acknowledgements This work was supported by the Kristian Gerhard Jebsen Foundation. Data was processed in part by using the super computer Stallo at the University of Troms $\varnothing$ (UiT) provided by Norwegian Metacenter for Computational Science (NOTUR) under the project uit-phys-014. The CESM experiments were performed at the super computer Vilje at Norwegian University of Science and Technology (NTNU) under the NOTUR project NN9348K. We are thankful to Dr. Gabriele Messori for the many useful discussions. We would also like to thank Dr. Benoit Vannière and three anonymous reviewers for their valuable suggestions to improve this work.
Open Access This article is distributed under the terms of the Creative Commons Attribution 4.0 International License (http:// creativecommons.org/licenses/by/4.0/), which permits unrestricted use, distribution, and reproduction in any medium, provided you give appropriate credit to the original author(s) and the source, provide a link to the Creative Commons license, and indicate if changes were made.

\section{References}

Bitz CM, Shell KM, Gent PR, Bailey DA, Danabasoglu G, Armour KC, Holland MM, Kiehl JT (2012) Climate sensitivity of the community climate model version 4. J Clim 25:3053-3070

Bjerknes J (1964) Atlantic air-sea interaction. Adv Geophys 10(1):82

Carleton AM (1988) Meridional transport of eddy sensible heat in winters marked by extremes of the north Atlantic oscillation, 1948/49-1979/80. J Clim 1(2):212-223

Czaja A, Robertson AW, Huck T (2003) The role of Atlantic oceanatmosphere coupling in affecting north Atlantic oscillation variability. The North Atlantic oscillation: climatic significance and environmental impact, pp 147-172

Dee D, Uppala S, Simmons A, Berrisford P, Poli P, Kobayashi S, Andrae U, Balmaseda M, Balsamo G, Bauer P et al (2011) The era-interim reanalysis: configuration and performance of the data assimilation system. Q J R Meteorol Soc 137(656):553-597

Gill AE (1982) International Geophysics, 30: Atmosphere-ocean Dynamics. Elsevier

Graversen RG (2006) Do changes in the midlatitude circulation have any impact on the Arctic surface air temperature trend? J Clim 19(20):5422-5438

Graversen RG, Kallen E, Tjernstrom M, Kornich H (2007) Atmospheric mass-transport inconsistencies in the era- 40 reanalysis. $Q$ J R Meteorol Soc 133(624):673-680

Heaviside C, Czaja A (2013) Deconstructing the Hadley cell heat transport. Q J R Meteorol Soc 139(677):2181-2189

Held IM (2001) The partitioning of the poleward energy transport between the tropical ocean and atmosphere. J Atmos Sci 58(8):943-948

Hurrell JW, Holland MM, Gent PR, Ghan S, Kay JE, Kushner P, Lamarque JF, Large WG, Lawrence D, Lindsay K et al (2013) The community earth system model: a framework for collaborative research. Bull Am Meteorol Soc 94(9):1339-1360

Marshall J, Kushnir Y, Battisti D, Chang P, Czaja A, Dickson R, Hurrell J, McCARTNEY M, Saravanan R, Visbeck M et al (2001) North Atlantic climate variability: phenomena, impacts and mechanisms. Int J Climatol 21(15):1863-1898

Messori G, Czaja A (2013) On the sporadic nature of meridional heat transport by transient eddies. Q J R Meteorol Soc 139(673):999-1008

Novak L, Ambaum MH, Tailleux R (2015) The life cycle of the north Atlantic storm track. J Atmos Sci 72(2):821-833

Oort AH, Peixóto JP (1983) Global angular momentum and energy balance requirements from observations. Adv Geophys 25:355-490

Oort AH, Yienger JJ (1996) Observed interannual variability in the Hadley circulation and its connection to ENSO. J Clim 9(11):2751-2767

Peixoto JP, Oort AH (1992) Physics of climate. American Institute of Physics, New York

Sun DZ (2000) The heat sources and sinks of the 1986-87 El Niño. J Clim 13(20):3533-3550

Sun DZ, Trenberth KE (1998) Coordinated heat removal from the equatorial Pacific during the 1986-87 El Nino. Geophys Res Lett 25(14):2659-2662 
Thompson DW, Barnes EA (2014) Periodic variability in the largescale southern hemisphere atmospheric circulation. Science 343(6171):641-645

Trenberth KE (1991) Climate diagnostics from global analyses: conservation of mass in ECMWF analyses. J Clim 4(7):707-722
Trenberth KE, Caron JM (2001) Estimates of meridional atmosphere and ocean heat transports. J Clim 14(16):3433-3443

Trenberth KE, Caron JM, Stepaniak DP (2001) The atmospheric energy budget and implications for surface fluxes and ocean heat transports. Clim Dyn 17(4):259-276 